\title{
Distribución espacial, estructura social y amenazas de conservación de una pequeña comunidad de delfines nariz de botella, Tursiops truncatus (Odontoceti: Delphinidae) en Ecuador
}

\author{
Fernando Félix ${ }^{1,2}$, Melanie Zavala ${ }^{3} \&$ Ruby Centeno ${ }^{4}$ \\ 1. Pontificia Universidad Católica del Ecuador (PUCE), Av. 12 de Octubre 1076, Quito, Ecuador. \\ 2. Museo de Ballenas. Av. Enríquez Gallo entre calles 47 and 50, Salinas, Ecuador; fefelix90@hotmail.com \\ 3. Urb. Santa Cecilia Mz 27 villa 1-3. Guayaquil, Ecuador; melanie_zavalav26@hotmail.com \\ 4. Time for English and Spanish School. Av. Circunvalación 906, Guayaquil, Ecuador; rubycenteno@hotmail.com
}

$$
\text { Recibido 20-XII-2018. C Corregido 30-V-2019. Aceptado 07-VIII-2019. }
$$

\begin{abstract}
Spatial distribution, social structure and conservation threats of a small community of bottlenose dolphins, Tursiops truncatus (Odontoceti: Delphinidae) in Ecuador. A resident community of bottlenose dolphins (Tursiops truncatus) was studied irregularly between 2005 and 2018 around the tip of the Santa Elena Peninsula, Ecuador $\left(2^{\circ} 11^{\prime} \mathrm{S} \& 81^{\circ} 0.7^{\prime} \mathrm{W}\right)$. Opportunistic sightings and systematic surveys from the beach and at sea were carried out along $40 \mathrm{~km}$ of coast, accounting for $917.2 \mathrm{~km}$ of tracking by car from land and 707.4 $\mathrm{km}$ of boat tracking by sea. Average group size was 5.31 dolphins/group ( $\mathrm{SD}=1.97$, range $1-10$ ), with no significant changes throughout the study period. From land, however, the group size was underestimated $32 \%$ on the average. This small bottlenose dolphin community currently has only nine individuals, including six adults, one immature and two calves, and is the smallest community within the Gulf of Guayaquil. The encounter rate ranged between 0.03 dolphins $/ \mathrm{km}$ in the northwestern part and 0.32 dolphins $/ \mathrm{km}$ in the South, where dolphins concentrate their activities, possibly because human activities are less intense there. Dolphins were generally distributed in the first $200 \mathrm{~m}$ from the shore, reaching up to $1200 \mathrm{~m}$ in the Northern shallower part and where port and tourist activities concentrate. The dolphins' speed was significantly higher when they were followed from a boat at close range $(5.87 \mathrm{~km} / \mathrm{h})$ than when they were monitored from the beach $(2.9 \mathrm{~km} / \mathrm{h})(\mathrm{P}<0.01)$, which suggests that boat tracking had an effect on dolphin's movements. Pairwise cluster analysis showed that animals from this community show high rates of association among each other (average 0.67, range 0.01-1.0), indicating that is a highly cohesive community. Dolphins also showed high level of residence (average occurrence index $=0.62$ ). During the study, two main threats were identified, a gillnet fishery in the Southwestern part and an intense fishing boat traffic in the Northwestern. Most of the study area is currently part of a coastalmarine protected area created in 2008, which offers an opportunity for the recovery and conservation of this dolphin community. Given its fragility, we recommend the environmental authorities to address potential threats for this dolphin community by eliminating gillnets, implementing an exclusion zone for fishing gear and boat traffic of $1 \mathrm{~km}$ width from the shore, and limit the speed of any type of vessel to $10 \mathrm{knots}$ within the reserve.
\end{abstract}

Key words: ecology; behavior; conservation and management; bottlenose dolphin; Tursiops truncatus; anthropogenic threats; Ecuador.

Félix, F., Zavala, M., \& Centeno, R. (2019). Distribución espacial, estructura social y amenazas de conservación de una pequeña comunidad de delfines nariz de botella, Tursiops truncatus (Odontoceti: Delphinidae) en Ecuador. Revista de Biología Tropical, 67(4), 1059-1076. 
El delfín nariz de botella (Tursiops truncatus, Montagu, 1821), nombre derivado de la traducción del inglés "bottlenose dolphin" pero conocido en Latinoamérica de diferentes formas según el país tales como bufeo, tonina, tursión, delfín mular, entre otros, es una especie de cetáceo menor que se distribuye a lo largo de los mares tropicales y templadas del mundo (Jefferson, Webber, \& Pitman, 2008; Hammond et al., 2012a). Actualmente hay una segunda especie reconocida en el género Tursiops: T. aduncus (Ehrenberg, 1633), un poco más pequeño y de distribución limitada al Pacífico occidental y Océano Índico (Hammond et al. 2012a, 2012b). Sin embargo, en el caso de T. truncatus, a todo lo largo de su área de distribución existe una cantidad aún no definida de formas o morfotipos asociados a ambientes costeros, estuarinos y marinos -probablemente como consecuencia de un proceso incipiente de especiación asociado a la explotación de nichos ecológicos distintos- que requieren más estudios taxonómicos para su apropiada caracterización (Van Waerebeek, Reyes, Read, \& McKinnon, 1990; Van Waerebeek et al., 2017; Sanino Van Waerebeek, Van Bressem, \& Pastene, 2005; Tezanos-Pinto et al., 2009; Perrin, Thieleking, Walter, Archer, \& Robertson, 2011; Fruet et al., 2016; Bayas-Rea, Félix, \& Montúfar, 2018). Al ecotipo que habita a lo largo de la línea de costa y aguas estuarinas interiores se lo conoce con el término en inglés "inshore", para diferenciarlo de otras formas costeras que estarían más relacionadas con el ecotipo oceánico y que correspondería a procesos recientes de colonización (Tezanos-Pinto et al., 2009; Natoli, Peddemors, \& Hoelzel, 2004).

Los delfines nariz de botella costeros viven en sociedades complejas llamadas fusiónfisión, esto es, sus miembros forman asociaciones temporales que se desarrollan generalmente dentro de un territorio bien definido (Wells, Scott, \& Irvine, 1987; Connor, Smolker, \& Richards, 1992; Lusseau et al., 2006). Esta forma de organización social ocurre en diferentes partes del mundo donde han sido estudiados como en Estados Unidos (Wells et al., 1987), Brasil (Simoes-Lopes \& Fabian, 1999) o Australia (Möller, Wiszniewski, Allen, \& Beheregaray, 2007). La organización social, distribución y patrones de movimiento están regulados por factores biológicos intrínsecos como el sexo y la edad (Félix, 1997; Fury, Rucksthul, \& Harrison, 2013; Morteo, RochaOlivares, \& Abarca-Arenas, 2014), factores ecológicos como la distribución y disponibilidad de sus presas, presión por depredación y competencia intra e interespecífica, así como condiciones ambientales cambiantes debido a la variabilidad estacional, al ciclo de mareas, entre otros, y en algunos casos en función de usos antrópicos de la zona costera que pueden llegar a modificar sus patrones espacio-temporales de distribución (e.g., Lusseau, 2004; Morteo, Rocha-Olivares, Arceo-Briseño, \& Abarca-Arenas, 2012a; Di Giacomo \& Ott, 2016). Al igual que otros delfines costeros alrededor del mundo, los delfines nariz de botella costeros están amenazados por actividades humanas como la pesca, el tráfico marítimo y la degradación del hábitat por la contaminación en sus diferentes formas (Parsons \& Jefferson, 2000; Reeves, Smith, Crespo, \& Notarbartolo di Sciara, 2003; Reeves, McClellan, \& Werner, 2013; Van Waerebeek et al., 2007; Azevedo et al., 2017).

En la costa oeste de Sudamérica, los delfines nariz de botella han sido registrados entre Colombia y el sur de Chile (Olavarría et al., 2010; Herrera et al., 2011), aunque no es claro si la distribución es continua (Van Waerbeek et al., 1990, 2017). En Ecuador la especie es conocida con el nombre local de "bufeo", del cual se han identificado dos ecotipos según su ámbito de distribución y características morfológicas (Van Waerebeek et al., 1990; Félix et al., 2018b). El bufeo del ecotipo costero es el cetáceo de distribución más costera en Ecuador, por lo general se le encuentra en los primeros $1000 \mathrm{~m}$ de la costa en grupos de 10 animales o menos, aunque en ocasiones pueden llegar a 50 (Félix, 1994). Estudios de la especie se han llevado a cabo desde la década de 1990 en el golfo de Guayaquil, suroeste de Ecuador, donde habita una población residente estructurada en subunidades o "comunidades", cuya 
organización social se basa en grupos familiares dominados por madres con crías, alrededor de los cuales otros individuos de la misma comunidad se relacionan con diferente nivel de afinidad según su clase de edad y sexo (Félix, 1997; Félix, Calderón, Vintimilla, \& BayasRea, 2017). Una de estas comunidades habita en el extremo norte del golfo de Guayaquil, en la punta de la península de Santa Elena, donde las condiciones ambientales son diferentes a las del resto del golfo por tener características más oceánicas, en contraste con las condiciones estuarinas que prevalecen en la parte interna y sur del golfo de Guayaquil, caracterizadas por aguas de baja salinidad y alta productividad (Stevenson, 1981) y ausencia de grandes depredadores. Por ello se esperaría que la estructura social de la comunidad de delfines de Santa Elena sea similar a las del resto del golfo, aunque con algún grado de diferenciación considerando las características fisiográficas y ecológicas del área.

Aunque la especie se considera a nivel global como de baja preocupación desde el punto de vista de su estado de conservación (Hammond et al., 2012a), los bufeos costeros de Ecuador presentan una alta tasa de cicatrices corporales de origen antrópico (Félix et al., 2018a), una alta prevalencia de enfermedades cutáneas probablemente asociadas a condiciones ambientales en degradación, particularmente en zonas estuarinas y de alta concentración urbana (Van Bressem et al., 2015) y tendencia poblacional decreciente (Félix et al., 2017), por ello localmente se los ha catalogado como vulnerables (Jiménez, Álava, Castro, Denkinger, \& Haase, 2011). Valorar su estado poblacional e identificar los problemas que afectan a estos animales y su vinculación con actividades antrópicas específicas es un primer paso para definir acciones de mitigación. Por ello los objetivos planteados en este estudio fueron evaluar aspectos tales como patrones de asociación entre animales, estructura social y uso de hábitat de la comunidad de bufeos costeros que habita alrededor de la península de Santa Elena para entender la dinámica poblacional e identificar las principales amenazas que podrían estar afectándolos. Al no existir información previa de esta comunidad de delfines, este estudio ofrece una línea base de conocimiento que ayudará a las autoridades ambientales a definir las mejores estrategias de gestión para regular las actividades humanas que se desarrollan en la zona y asegurar la viabilidad de la esta población en el largo plazo.

\section{MATERIALES Y MÉTODOS}

Área de estudio: El área de estudio se localiza en la zona más occidental de la península de Santa Elena y de Ecuador hacia el océano Pacífico, conocida como la puntilla $\left(2^{\circ} 11^{\prime} \mathrm{S}\right.$ $\& 81^{\circ} 0^{\prime} \mathrm{W}$ ) (Fig. 1). La zona costera se caracteriza por una sucesión de playas estrechas extensas y acantilados de baja altura. Al sur de la puntilla, las condiciones de la zona costera son más extremas que al lado norte pues la península está orientada hacia el noroeste de manera perpendicular a los vientos prevalentes del suroeste. En contraste, la zona norte consiste de bahías protegidas con oleaje bajo o moderado, donde se concentran las actividades humanas (Instituto Nazca, 2008).

En la zona de estudio se encuentran tres ciudades colindantes: Santa Elena, Libertad y Salinas, con una población de 308000 habitantes (INEC, 2017). Salinas es el sitio turístico más importante de la costa de Ecuador con un turismo estacional de tipo masivo que se extiende entre enero y abril y otra temporada menos intensa entre julio y agosto. En Salinas, están localizados dos importantes puertos pesqueros artesanales, Santa Rosa y Anconcito, con 2010 embarcaciones entre los dos (Herrera, Castro, Coello, Saa, \& Elías, 2013), y cuenta con dos marinas para yates privados. Otras actividades alrededor del área de estudio incluyen la producción de larvas de camarón, extracción de sal y de petróleo en la zona costera. Además, en la Puntilla de Santa Elena están ubicadas 3 bases militares y un aeropuerto. En Libertad está la segunda refinería de petróleo del país y además es puerto pesquero. Libertad es el municipio de mayor densidad poblacional del país con 3840 $\mathrm{hab} / \mathrm{km}^{2}$ (Hurtado, Hurtado, \& Hurtado, 2012). 


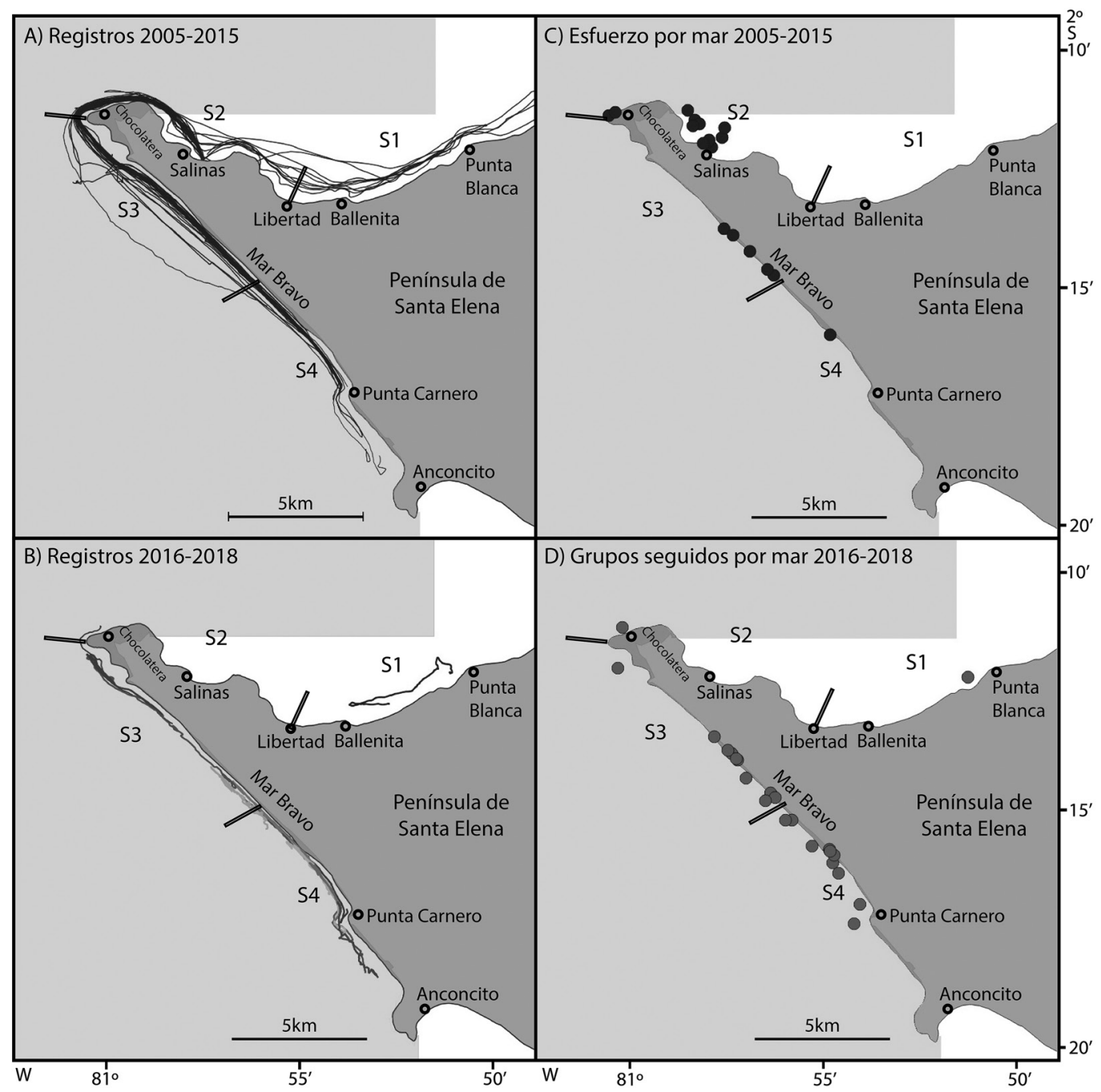

Fig. 1. Esfuerzo dedicado por mar y ubicación de los registros de delfines alrededor de la puntilla de Santa Elena: A) recorridos en el mar en el período 2016-2018; B) seguimiento de los delfines en el mar; C) sitios de registro de grupos de delfines en el período 2005-2015; D) sitios de registro de delfines en el período 2016-2018. En el mapa se indica además la ubicación de los cuatro sectores en que se dividió el área de estudio (S1-S4) separados por líneas negras perpendiculares a la costa. La parte gris claro corresponde al área protegida Puntilla de Santa Elena. El área protegida también incluye parte de la zona costera.

Fig. 1. Dedicated effort by sea and location of the records of dolphins around Santa Elena tip: A) surveys at sea in the period 2016-2018; B) tracking dolphins at sea; C) dolphin group sightings in the period 2005-2015; D) dolphin group sightings in the period 2016-2018. The map also indicates the location of the four sectors into which the study area was divided (S1S4) separated by black lines perpendicular to the coast. Light gray area corresponds to the Puntilla de Santa Elena marine protected area. The protected area also includes part of the coastal zone. 
Santa Elena está un poco más hacia el interior y las actividades costeras se limitan al comercio, turismo y pesca artesanal. Los problemas ambientales de la zona incluyen una alta contaminación microbiológica producto de una baja cobertura de saneamiento y por las descargas crudas de aguas residuales de las plantas procesadoras de pescado, derrames operacionales de petróleo, baja tasa de cobertura de recolección de basura sólida y desarrollo urbano (Hurtado et al., 2012).

En el 2008, se creó en el extremo de la península de Santa Elena la "Reserva de Producción Faunística Marino Costera Puntilla de Santa Elena", con una extensión de 52231 ha de mar y 203 ha de zona terrestre (MAE, 2012). $\mathrm{El}$ área marina se extiende hasta $7.4 \mathrm{~km}$ alrededor de la punta de la península y $17 \mathrm{~km}$ hacia el sur a lo largo de la costa.

Observaciones y monitoreos: Entre marzo 2005 y marzo 2018 se monitoreó una comunidad de bufeos costeros alrededor de la puntilla de Santa Elena de manera no sistemática. Los registros incluyeron observaciones oportunistas desde la playa a lo largo de Mar Bravo, la Chocolatera y el muelle de Salinas y a bordo de embarcaciones de observación de ballenas entre 2005 y 2015 durante la temporada de reproducción de las ballenas jorobadas (junio-septiembre) (Fig. 1). Adicionalmente, se llevaron a cabo monitoreos dirigidos entre enero 2016 y marzo 2018 mediante recorridos en automóvil tipo sedán a una velocidad de $10-20 \mathrm{~km} / \mathrm{h}$ por $9.5 \mathrm{~km}$ en Mar Bravo entre Punta Carnero y el aeropuerto de Salinas. En esta zona la carretera se ubica a lo largo de la playa a una altura entre 1 y $3 \mathrm{~m}$ sobre el nivel de la marea alta. En el vehículo iba un conductor y dos observadores, uno en el asiento del copiloto y uno en el asiento trasero. El observador del asiento trasero usó binoculares $10 \mathrm{x}$ 50 durante la mayor parte de los recorridos. En cada viaje se hicieron entre 4 y 6 recorridos por la misma zona hasta ubicar un grupo de delfines. Una vez ubicados los delfines, dos observadores se bajaron del vehículo y siguieron a los delfines a pie a lo largo de la playa para fotografiar sus aletas dorsales y registrar información del evento. Si el grupo se movía más rápido que los investigadores entonces se retomaba el recorrido en automóvil, haciendo tantas paradas como fuera necesario para seguir registrando el movimiento de los animales hasta que se perdían de vista.

Entre julio 2016 y marzo 2018 se realizaron adicionalmente recorridos de entre 43 y $82 \mathrm{~km}$ en bote alrededor de la puntilla, por el sur hasta Anconcito y por el norte hasta Punta Blanca, usando como puerto Salinas (Fig. 1). Para el efecto se usó un bote de $6 \mathrm{~m}$ de longitud equipado con un motor fuera de borda de 48 HP, en el cual se recorrieron los sitios donde previamente se hicieron las observaciones no sistemáticas tanto desde la playa como a bordo de botes de turismo. Usualmente, se recorrió primero el lado sur del área de estudio y si no se encontraron delfines en esta zona se avanzó a la parte norte. Los recorridos siguieron una ruta paralela a la costa a $100-300 \mathrm{~m}$ de la orilla en la zona sur y fueron un poco más variables y separados de la orilla en la zona norte debido a la poca profundidad y a las actividades portuarias en Salinas y Libertad. Al menos dos observadores participaron en cada viaje buscando los delfines a simple vista. Tanto las observaciones desde tierra como de seguimiento en el mar se hicieron en condiciones oceánicas Beaufort $<3$.

La información recolectada durante los avistamientos en automóvil y en bote incluyó: posición geográfica, tamaño y composición de los grupos por clases de edad y sexo, estado comportamental (alimentación, descanso, tránsito o socialización) y dirección del grupo observado. La identificación de los animales por clases de edad se hizo con base al tamaño relativo de los animales medidos a simple vista en el campo (Félix, 1997). Los animales más grandes fueron identificados como adultos, los animales más pequeños pero no evidentemente asociados a otro adulto como su potencial madre fueron clasificados como subadultos o inmaduros. Finalmente, aquellos animales que midieron entre $1 / 3$ y $1 / 2$ del tamaño de un adulto y estaban evidentemente asociados a 
un adulto, presumiblemente la madre, fueron catalogados como crías.

Cámaras fotográficas digitales (6-24 megapixeles) con lentes de 70-300 $\mathrm{mm}$ y $100-400$ $\mathrm{mm}$ fueron utilizadas para tomar fotografías de las aletas dorsales y otras partes corporales. Las aletas y otras marcas observadas en las fotografías fueron utilizadas para identificar individuos mediante la presencia de muescas en el borde posterior de las aletas dorsales (véase trabajos previos Félix, 1997; Félix et al., 2017). Las fotografías de mejor calidad con respecto a enfoque y ángulo perpendicular fueron utilizadas para crear un catálogo que fue actualizado regularmente. A cada individuo le fue asignado con un código alfanumérico de identificación. Las fotografías también fueron útiles para registrar comportamientos y otras características individuales específicas tales como infecciones cutáneas y cicatrices (Van Bressem et al., 2015; Félix et al., 2018a). Durante los períodos de seguimiento, tanto desde tierra como desde el mar, se georreferenciaron los avistamientos y recorridos utilizando un GPS Garmin ${ }^{\circledR}$ 64. Esta información fue utilizada posteriormente para estimar el esfuerzo de muestreo.

La información de los monitoreos dirigidos fue utilizada para calcular la tasa de encuentro (delfines $/ \mathrm{km}$ de recorrido). La velocidad mínima del grupo de delfines se estimó utilizando como distancia total la longitud del tramo de seguimiento del grupo de delfines dividido para el tiempo total que duró el avistamiento. Esto ocurrió tanto en el caso de los monitoreos en bote donde los grupos de defines fueron seguidos a corta distancia $(20-50 \mathrm{~m})$ como en automóvil, donde la referencia de la distancia recorrida fue una línea imaginaria perpendicular entre el grupo de delfines y el automóvil. Para evaluar el uso de hábitat con más detalle, el área de estudio fue dividida en cuatro sectores de aproximadamente $10 \mathrm{~km}$ de longitud, dos en la zona norte (sectores 1 y 2) y dos en la zona oeste y sur (sectores 3 y 4) (Fig. 1). En los sectores 1 y 2 se ubican las zonas urbanas, por lo que hay una intensa actividad antrópica en la costa y el mar (Salinas, Libertad, Ballenita,
Punta Blanca). El sector 1 se caracteriza por acantilados bajos y de mediana altura en el sur y playas arenosas en el norte. El sector 2 se caracteriza por bahías con fondos arenosos en la parte central y acantilados bajos y fondos rocosos en la parte oeste (Chocolatra). Los sectores 3 y 4 están orientados a sotavento, por lo cual en la zona costera hay un fuerte oleaje, particularmente durante el segundo semestre del año cuando los vientos alisios son fuertes. Hay dos bahías en la parte norte (sector 3) con acantilados bajos y una mezcla de fondos arenosos y rocosos, pero en la parte centro y sur (Mar Bravo) el fondo es arenoso y se extiende hasta más allá de la mitad del sector 4 (Punta Carnero). A partir de allí, la costa se vuelve rocosa, con bajos que quedan descubiertos en bajamar, algunos de los cuales se ubican varios cientos de metros de la orilla. Hacia el sur de Punta Carnero nuevamente la costa se vuelve arenosa (Fig. 1).

Los recorridos, grabados con el GPS, fueron cortados con la herramienta corte del software MapSource de Garmin ${ }^{\circledR}$ para separar la parte correspondiente al esfuerzo de búsqueda del período de seguimiento de los grupos. Esta información fue analizada mediante el software QGIS (2016) para elaborar mapas. La distancia de los grupos hasta la costa también fue estimada con la herramienta medición de QGIS.

El monitoreo incluyó la identificación de potenciales amenazas para esta comunidad de delfines. Dado el corto período de esfuerzo dedicado, esta parte del trabajo se enfocó en la cuantificación de embarcaciones pesqueras faenando en la zona de estudio durante los recorridos. Para la evaluación de otras amenazas como el tráfico marítimo, particularmente de embarcaciones de pesca artesanal, se utilizó información publicada disponible con respecto al tamaño de las flotas pesqueras de Santa Rosa (Salinas) y Anconcito (Herrera et al., 2013).

Estructura social: La estructura social se evaluó a través de un análisis clúster jerárquico usando el índice de medio-peso (Cairns \& Schwager, 1987) implementado en el programa SocProg (Whitehead, 2009). El índice estima 
el nivel de asociación entre pares de individuos con base al número de veces que ambos individuos fueron vistos como parte del mismo grupo. Tiene un valor entre 0 y 1 , donde 0 indica que los animales nunca fueron vistos juntos y 1 indica que los animales fueron encontrados siempre juntos. Los valores de asociación se presentan como un dendrograma o diagrama de árbol con la identidad de los individuos en el eje vertical y el nivel de asociación en el eje horizontal. El análisis se restringió al período 2014-2018 para reducir el sesgo temporal e incluyó animales que fueron vistos al menos tres veces.

\section{Definiciones:}

Grupo. Un grupo de delfines se definió como todos aquellos animales que fueron observados durante el período de avistamiento, por lo general realizaron actividades similares y se movieron como una unidad con similar velocidad y misma dirección (Félix et al., 2017). El número de animales en un grupo fue determinado por conteos directos de los animales identificados (monitoreos dirigidos) o estimado como un promedio cuando no se pudieron identificar todos los animales (conteos desde tierra).

Comunidad. Término que se lo usa en un contexto social para definir a la unidad poblacional que reside en el área de estudio. Esta definición se basa en Wells et al. (1987), quienes definieron como comunidad al grupo de delfines que habita un área y cuyo nivel de asociación entre sus miembros es más alto que con animales de áreas vecinas.

Fidelidad al área de estudio. Se estimó mediante el índice de ocurrencia (IO), determinado por el número de veces en las cuales los individuos fueron vistos en el total de ocasiones de recaptura posibles en un período determinado (Morteo, Rocha-Olivares, \& Morteo, 2012b). Un valor de 1 indica que un animal fue visto siempre en cada ocasión.

Estado de comportamiento. Describe el comportamiento de los delfines con base a descripciones previas en la especie (Shane, 1990):
1. Descanso/merodeo. Período en que los delfines se movieron dentro de un área pequeña generalmente con una velocidad promedio menor a $5 \mathrm{~km} / \mathrm{h}$.

2. Tránsito. Cuando los delfines tuvieron una dirección continua y mantuvieron una velocidad constante generalmente > $5 \mathrm{~km} / \mathrm{h}$.

3. Alimentación. Período en que se observaron movimientos irregulares, con nados rápidos, buceos prolongados que iniciaban generalmente con levantamiento de cola o períodos en que los delfines lanzaron peces sobre el agua. En ocasiones durante estos períodos se observaron peces saltar cerca de los delfines y aves marinas volando alrededor.

4. Socialización. Periodos en que se observó a algunos miembros del grupo mostrando actividad de superficie como saltos, nados rápidos, persiguiéndose o con entrerozamientos.

Análisis estadísticos: Dos tipos de análisis estadísticos fueron realizados con los datos obtenidos en este estudio: la tabla de contingencia de Chi cuadrada para comparar proporciones de clases grupales y la prueba de $t$ para dos muestras asumiendo varianzas desiguales para comparar variables como el tamaño y velocidad del grupo, previo a la trasformación logarítmica de los datos. Los test estadísticos se realizaron con el complemento análisis de datos incluido en Microsoft Excel®.

\section{RESULTADOS}

Esfuerzo: Durante el período de muestreo de manera oportunista (2005-2015) se registraron 18 grupos, ocho desde la playa y diez en el mar (Tabla 1). En el período de esfuerzo dirigido (enero 2016-marzo 2018) se hicieron 16 recorridos en automóvil por un total de $764 \mathrm{~km}$, en los cuales se registraron 14 grupos (Tabla 2), y 14 viajes en bote en los que se recorrió 707 $\mathrm{km}$ durante $63.7 \mathrm{~h}$ de navegación, incluyendo $21.6 \mathrm{~h}$ con los animales, período en el que se 
TABLA 1

Grupos, número de animales e individuos identificados durante los encuentros de oportunidad desde la playa y en bote. Período 2005-2015

TABLE 1

Groups, number of animals and individuals identified during the opportunity sightings from the beach and by boat. Period 2005-2015

\begin{tabular}{|c|c|c|c|}
\hline Variable & Desde la playa & En el mar & Total \\
\hline Grupos & 8 & 10 & 18 \\
\hline Total delfines & 37 & 58 & 95 \\
\hline Tamaño promedio de grupo & $4.6(\mathrm{SD}=1.09)$ & $5.8(\mathrm{SD}=1.61)$ & $5.2(\mathrm{SD}=2.19)$ \\
\hline Delfines identificados & 17 & 6 & $17 *$ \\
\hline
\end{tabular}

*Número total de animales diferentes.

TABLA 2

Información del esfuerzo dedicado mediante recorridos en auto desde la playa e información general de los grupos observados (Sectores 3 y 4). Período: enero-junio 2016. Solo los sectores 3 y 4 fueron monitoreados por este método

TABLE 2

Information on the effort made by car trips along the beach and general information of the recorded groups (Sectors 3 and 4). Period: January-June 2016. Only sectors 3 and 4 were monitored by this method.

\begin{tabular}{lccc}
\multicolumn{1}{c}{ Variable } & Sector 3 & Sector 4 & TOTAL \\
Esfuerzo total $(\mathrm{km})$ & 232 & 531 & 764 \\
Grupos & 6 & 8 & 14 \\
Total delfines & 20 & 42 & 62 \\
Tamaño promedio de grupo & $3.3(\mathrm{SD}=1.3)$ & $5.1(\mathrm{SD}=1.7)$ & $4.1(\mathrm{SD}=1.8)$ \\
Delfines identificados & 7 & 9 & $9^{*}$ \\
Tasa de encuentro $($ delfines $/ \mathrm{km})$ & 0.086 & 0.079 & 0.081 \\
Distancia de seguimiento $(\mathrm{km})$ & 12.3 & 14.2 & 26.5 \\
Tiempo de seguimiento $(\mathrm{h})$ & 4.45 & 4.42 & 8.87 \\
Velocidad de delfines $(\mathrm{km} / \mathrm{h})$ & 2 & 3.2 & 2.9 \\
\hline
\end{tabular}

*Número total de animales diferentes.

registraron 12 grupos más (Tabla 3) (Fig. 1A y Fig. 1B).

Tamaño y composición grupal: El tamaño promedio de los grupos fue de 5.31 delfines/grupo ( $\mathrm{DE}=1.97$, rango: 1-10 animales). Los grupos más grandes fueron encontrados durante los recorridos dirigidos en bote (6.7 delfines/grupo, $\mathrm{DE}=0.45, \mathrm{~N}=12$ ) y los más pequeños durante los avistamientos dirigidos desde tierra (4.14 delfines/grupo, $\mathrm{DE}=1.84$, $\mathrm{N}=14$ ). En general, los grupos observados desde el mar fueron significativamente más grandes (32\% en promedio) que los grupos observados desde tierra (prueba de $t=2.88$,
$\mathrm{P}<0.01$ ), lo cual indica que desde tierra el tamaño de los grupos fue subestimado. La proporción de adultos varió entre 71.4 y 76.3 \% y la proporción de crías entre 28.6 y $21.1 \%$ en los grupos avistados en el mar y desde tierra respectivamente, diferencias que no resultaron estadísticamente significativo $\left(\mathrm{X}^{2}=0.93, \mathrm{P}>\right.$ $0.05)$. Adicionalmente, un subadulto fue observado desde tierra $(2.6 \%)$.

Con respecto a posibles cambios en la estructura grupal en el tiempo, no se encontraron diferencias significativas en el tamaño promedio de los grupos observados de forma oportunista $(5.2, \mathrm{SD}=2.19)$ y mediante muestreos dirigidos desde tierra $4.1(\mathrm{SD}=1.8)$ 
TABLA 3

Esfuerzo dedicado en bote e información de los grupos seguidos. Periodo: julio 2016-marzo 2018

TABLE 3

Effort dedicated by boat and information on the followed groups. Period: July 2016-March 2018

\begin{tabular}{lccccc}
\multicolumn{1}{c}{ ITEM } & Sector 1 & Sector 2 & Sector 3 & Sector 4 & TOTAL \\
Esfuerzo total $(\mathrm{km})$ & 81.2 & 206.7 & 245.8 & 173.7 & 707.4 \\
Grupos & 1 & 1 & 2 & 8 & 12 \\
Total delfines & 7 & 7 & 12 & 55 & 81 \\
Tamaño grupal promedio & $7(\mathrm{SD}=0)$ & $7(\mathrm{SD}=0)$ & $6(\mathrm{SD}=0)$ & $7(\mathrm{SD}=0)$ & $6.8(\mathrm{SD}=0.35)$ \\
Delfines identificados & 7 & 7 & 6 & 7 & $7 *$ \\
Tasa de encuentro $(\mathrm{del} . / \mathrm{km})$ & 0.086 & 0.034 & 0.049 & 0.317 & 0.15 \\
Distancia de seguimiento $(\mathrm{km})$ & 7.8 & 1.3 & 38.4 & 79.1 & 126.6 \\
Tiempo de seguimiento $(\mathrm{min})$ & 97.6 & 10.8 & 339.7 & 846 & 1294.1 \\
Velocidad de delfines $(\mathrm{km} / \mathrm{h})$ & 4.80 & 7.22 & 6.78 & 5.61 & 5.87 \\
\hline
\end{tabular}

*Número total de animales diferentes.

(prueba de $t=1.17, \mathrm{P}=0.25$ ), ni entre los grupos encontrados de forma oportunista y muestreos dirigidos en el mar $(6.8, \mathrm{SD}=0.35)$ (Tabla 1, Tabla 2 y Tabla 3) (prueba $t=-1.40, \mathrm{P}$ $=0.19$ ). Esto sugiere que ha habido estabilidad en el tamaño de los grupos desde que se iniciaron los registros en 2005.

Distribución y tasa de encuentro: Grupos de delfines fueron encontrados en la mayor parte de la zona de estudio (Fig. 1). Durante la etapa de muestreo de oportunidad (2005-2015), los avistamientos se realizaron principalmente en el sector 2 frente a Salinas y en el sector 3 de Mar Bravo (Fig. 1C). Sin embargo, durante los muestreos dirigidos (2016-2018) los grupos fueron encontrados con mayor frecuencia en la zona de Mar Bravo, principalmente en el sector 4 (Fig. 1D), y ninguno frente a Salinas, a pesar de que Salinas fue el puerto de salida de los muestreos dirigidos por mar.

La tasa de encuentro promedio durante los recorridos por tierra en vehículo fue de 0.081 delfines $/ \mathrm{km}$, siendo ligeramente más alta en el sector 3 que en el 4 (Tabla 2). La tasa de encuentro promedio durante los muestreos dirigidos por mar fue de 0.15 delfines $/ \mathrm{km}$ (rango 0.034-0.317 delfines $/ \mathrm{km}$ ), esto es, $85 \%$ mayor que en los muestreos dirigidos por tierra, siendo más abundantes en el sector 4 y menos abundantes en el sector 2 (Tabla 3).

Con respecto a la distancia de los grupos a la playa, en el sector 1 (Ballenita) los delfines se distribuyeron entre 800 y 1200 m de distancia de la orilla. En la parte sur (sectores 3 y 4), los delfines se movieron en los primeros 200 m desde la playa, pero en Punta Carnero y la Puntilla se alejaron entre 500 y $760 \mathrm{~m}$ de la orilla (ver recorridos en la Fig. 1A y Fig. 1B).

Uso de hábitat: La caracterización del uso de hábitat se hizo con base en 27 avistamientos realizados entre 2016 y 2018 (esfuerzo dirigido) (Fig. 2). En los sectores 1 y 2 solo se realizaron uno y dos avistamientos respectivamente durante este período, indicando que actualmente son zonas de poco uso. En el sector 1 se observaron durante el mismo avistamiento tres estados de comportamiento diferentes, descanso/merodeo, tránsito y alimentación. En el sector 2 se registraron solo dos, tránsito y descanso/merodeo. En contraste, en los sectores 3 y 4 se observaron los cuatro estados de comportamiento, aunque con diferente proporción; en el sector 3 hubo más períodos de alimentación y en el sector 4 más períodos de descanso/ merodeo, pero las diferencias no resultaron significativas $\left(\mathrm{X}^{2}=2.5\right.$; g.l. $\left.=3 ; \mathrm{P}>0.05\right)$, por 


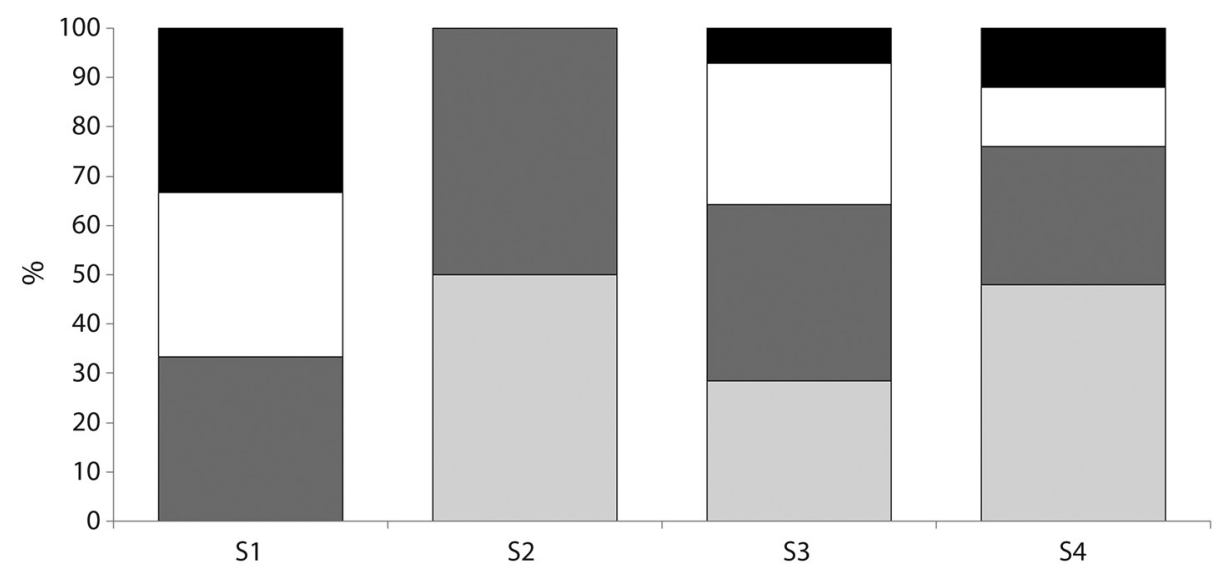

Fig. 2. Proporción de registro de los cuatro estados comportamentales de los grupos de delfines en cada sector del área de estudio alrededor de la península de Santa Elena. Período 2016-2018. Socialización (negro), alimentación (blanco), tránsito (gris oscuro) y descanso/merodeo (gris claro).

Fig. 2. Proportion of the four behavioral states recorded in the groups of dolphins in each sector of the study area around the Santa Elena peninsula. Period 2016-2018. Socialization (black), feeding (white), transit (dark gray) and resting/turns (light gray).

lo que se presume que los delfines usan los sectores 3 y 4 con los mismos fines.

Con respecto al tiempo que pasaron los grupos en cada sector durante los recorridos de esfuerzo dirigido, hubo algunas diferencias si los grupos fueron seguidos por tierra o por mar. En el período del esfuerzo dirigido desde tierra, los delfines fueron seguidos durante 4.45 $\mathrm{h}$ en el sector 3 y $4.42 \mathrm{~h}$ en el sector 4 , es decir aproximadamente $50 \%$ del tiempo en cada sector monitoreado (Tabla 2). En el caso de los recorridos por mar, el tiempo con los delfines en cada sector se distribuyó de la siguiente forma: $1.62 \mathrm{~h}$ en el sector $1(7.5 \%), 0.18 \mathrm{~h}$ en el sector $2(10.8 \%), 5.61 \mathrm{~h}$ en el sector 3 (26.4 $\%)$ y $14.1 \mathrm{~h}$ en el sector 4 (65\%).

La velocidad de los delfines durante los recorridos en bote fue significativamente más alta que durante los recorridos desde la playa, $5.87 \mathrm{~km} / \mathrm{h}$ y $2.9 \mathrm{~km} / \mathrm{h}$, respectivamente (prueba de $\mathrm{t}=4.85$, $\mathrm{P}<0.01$ ) (Tabla 2 y Tabla 3 ), lo cual sugiere que el seguimiento en bote tuvo un impacto sobre el movimiento de los delfines. En los grupos seguidos desde tierra la velocidad más alta se registró en el sector 4 y la más baja en el sector 3 (Tabla 2) y en los grupos seguidos por bote, las velocidades más altas se registraron en los sectores 2 y 3 , y la más baja en el sector 4.

En resumen, nuestros resultados sugieren que los sectores 3 y 4 en la parte sur de la puntilla son los más usados actualmente por los delfines de esta comunidad. Ocasionalmente los delfines se mueven hacia la parte norte en el sector 1, posiblemente para explotar otras áreas de alimentación. El sector 2 parece ser usado principalmente para tránsito entre los sectores 1 y 3 , pues es donde se registró la menor tasa de encuentro y la mayor velocidad de los delfines.

Tamaño de la comunidad, estructura social y fidelidad al sitio: A lo largo de los 13 años de seguimiento de esta comunidad de delfines se han identificado 21 animales diferentes, aunque en los últimos ocho años (julio 2010 a marzo 2018) solo se registraron 11. De ellos tres delfines fueron registrados por diez o más años (Nos. S4, S6 y S10) y observados de manera recurrente entre 14 y 35 veces (Fig. 3). Otros diez animales fueron vistos entre dos y cuatro años y siete animales se los observó una sola vez. De los animales registrados una solo vez, seis lo fueron antes de 2010 y uno en 2016 (S24). Los grupos de delfines fueron vistos en 


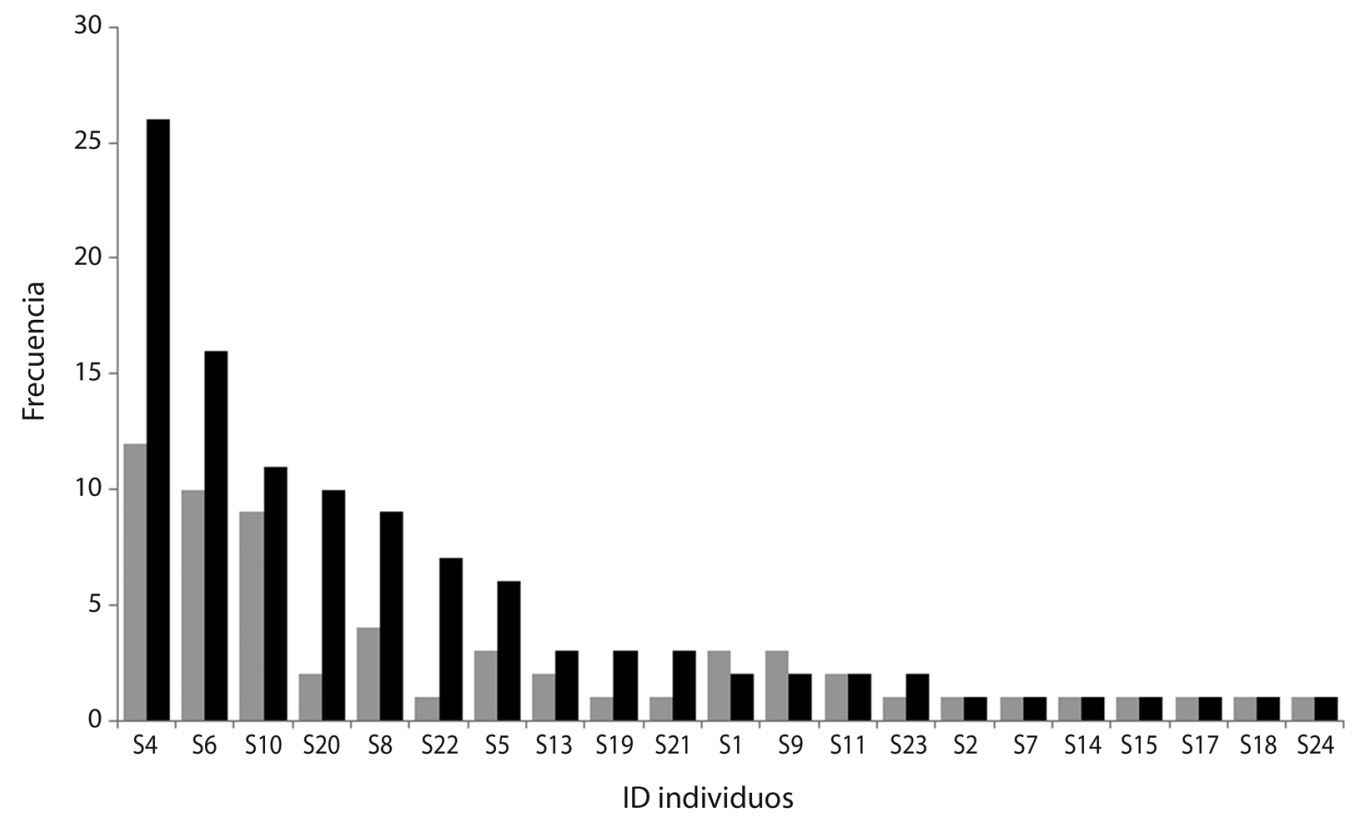

Fig. 3. Número de registros (barras negras) y tiempo de seguimiento en años (barras grises) de cada uno de los 21 individuos registrados en el área de estudio Santa Elena entre 2005 y 2018.

Fig. 3. Number of records (black bars) and follow-up time in years (gray bars) of each of the 21 individuals recorded in the study area between 2005 and 2018 .

10 de los 12 meses del año, con mayor número de registros en abril $(\mathrm{n}=8)$ y agosto $(\mathrm{n}=12)$. $\mathrm{El}$ individuo S8, un macho adulto de $3 \mathrm{~m}$ de longitud, fue observado durante cuatro años antes de ser encontrado muerto en marzo 2010.

Durante los recorridos en bote se registraron siete individuos diferentes mientras que en el período de estudio dirigido desde la playa se lograron identificar nueve. Así, entre julio 2016 y marzo 2018, esta comunidad de delfines estaría compuesta por solo nueve individuos: seis adultos, que incluyen dos hembras maduras que han tenido crías entre 2015 y 2016 (Nos. S10 y S20), dos probables machos adultos que fueron observados por mayor tiempo y que nunca se los vio asociados con crías (Nos. S4 y S6), dos adultos de sexo no conocido (Nos. S19 y S24), un subadulto (No. S21) y dos crías (Nos. S22 y S23). El índice de ocurrencia (IO) promedio estimado para esta comunidad de delfines durante el período de muestreo dirigido (2016-2018) fue de 0.62 (DS $=0.31$, rango $=0.045-0.90)$. Cinco de los seis adultos de esta comunidad de delfines mostraron valores de IO superiores a 0.72 , lo que sugiere una alta fidelidad al sitio.

El análisis clúster muestra que la comunidad de delfines de Santa Elena es altamente cohesionada, con un coeficiente de asociación promedio de 0.67 (Fig. 4). Con excepción del subadulto S21, los demás animales tuvieron coeficientes de asociación mayores a 0.77 . Las hembras adultas (S10 y S20) tuvieron el más alto coeficiente de asociación entre sí (1) pues siempre se las registró juntas.

Amenazas identificadas: Durante el período de investigación se identificaron dos amenazas principales para los bufeos costeros: 1) la presencia de una pesquería con redes agalleras de monofilamento de nylon de 12.5-15 $\mathrm{cm}$ de ancho de malla y alrededor de $1.5 \mathrm{~km}$ de longitud para capturar robalo (Centropomus 


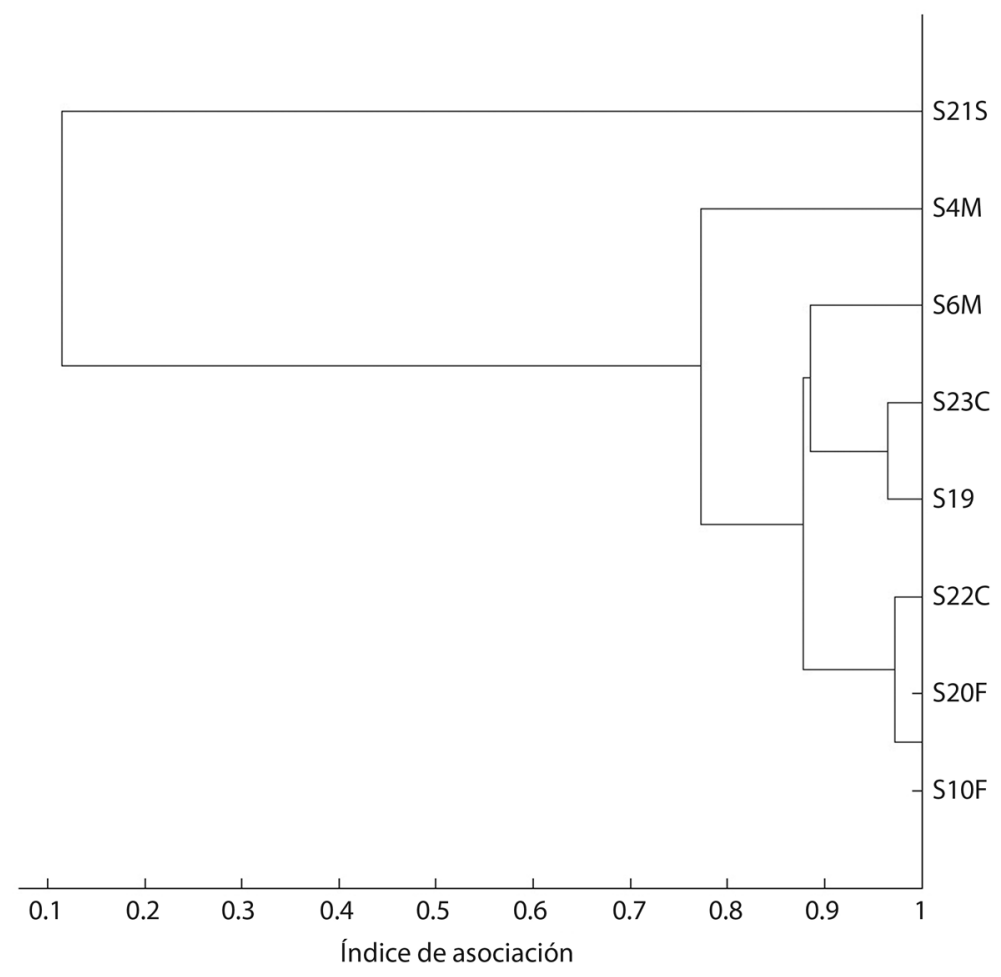

Fig. 4. Análisis clúster jerárquico de los delfines nariz de botella de la puntilla de Santa Elena. Período 2016-2018. Períodos de muestreo $=20$. Códigos de identidad (eje vertical) indican además el tipo de animal: $\mathrm{M}=$ macho, $\mathrm{F}=$ hembra, $\mathrm{S}=$ subadulto, $\mathrm{C}=$ cría.

Fig. 4. Hierarchical clustering analysis of the Santa Elena bottlenose dolphins. Period 2016-2018. Sampling Periods $=20$. Identity codes (the vertical axis), also indicate the type of animal: $\mathrm{M}=$ male, $\mathrm{F}=$ female, $\mathrm{S}=$ subadult, $\mathrm{C}=$ calf.

sp.), llamadas redes robaleras, a lo largo de la zona costera de Mar Bravo (sectores 3 y 4); y 2) un intenso tráfico de botes pesqueros artesanales de Santa Rosa, y en menor grado de botes de turismo, pesca deportiva, barcos cerqueros y tanqueros, en la zona norte (sector 3). En el primer caso se contaron hasta 20 botes pesqueros en un solo día, algunos de ellos colocando las redes muy cerca de la orilla y confirmando que hay un traslape con la distribución de los delfines.

En el caso del tráfico marítimo, tomando en cuenta solo la flota artesanal de Santa Rosa que tiene alrededor de 1500 embarcaciones, y considerando un promedio de dos días de duración por viaje, se estima que alrededor de 750 embarcaciones entran y salen del puerto cada día. Esto equivale en promedio a un bote que entra o sale cada minuto de este puerto. Si se considera además que el movimiento del puerto no es continuo durante el día, sino que se incrementa en las primeras horas en la mañana por el arribo masivo de botes con pesca y en horas de la tarde cuando dejan puerto para ir de pesca, el tráfico en las horas pico podría llegar a ser muy intenso. Adicionalmente, los botes pesqueros al salir de pesca se dirigen principalmente al oeste, bordeando la puntilla (S2), por lo que el tráfico de botes de pesca se concentra principalmente en esta zona.

\section{DISCUSIÓN}

La información obtenida en este estudio muestra que el tamaño de la comunidad de 
bufeos costeros que habita alrededor de puntilla de Santa Elena es mucho más pequeña comparada con otras comunidades mejor estudiadas en Ecuador (golfo de Guayaquil), que tienen entre 43 y 65 animales (Félix et al., 2017). Esta comunidad tiene actualmente nueve animales, parece bastante cohesionada y muestra un alto nivel de fidelidad al área a través del tiempo, como es típico en esta especie (Wells et al., 1987; Félix, 1997) y en otros cetáceos con similar estructura social (e.g., Bigg, Olesiuk, Ellis, Ford, \& Balcomb, 1990; Dungan, Wang, Araújo, Yang, \& White, 2016). No obstante, sin información histórica no es posible establecer con seguridad si su reducido número se debe a factores antrópicos o a la capacidad natural de la zona para sostener una población mayor de delfines. Nuestros datos más antiguos se remontan a algo más de una década atrás y sugieren que esta comunidad de delfines probablemente ha mantenido similar número de animales en los últimos 13 años. Es decir, si hubo un impacto por actividades humanas, éste debió haber ocurrido antes de este estudio, a menos que se trate de una colonización reciente, pero esto último parece menos probable debido a que la especie tiene una distribución continua a lo largo de la costa de Ecuador.

Aunque los bufeos costeros pueden vivir en comunidades semicerradas relativamente pequeñas, hay pocos antecedentes de comunidades tan reducidas como la de la puntilla de Santa Elena. Uno de estos casos ocurre en el estuario Tramandaí, al sur de Brasil, donde una comunidad residente de nueve animales ha sido monitoreada por años aparentemente sin cambios en el número total de animales (Di Giacomo \& Ott, 2016). Comunidades de bufeos costeros tan pequeñas son difíciles de estudiarlas, probablemente son remanentes de comunidades de delfines que en algún momento fueron más grandes y han sufrido reducciones considerables y podrían estar en proceso de extinción. Es importante destacar que, a pesar de su reducido tamaño, tanto la comunidad de Tramandí como de Santa Elena mantienen la capacidad de reproducción, como lo demuestra la presencia de crías, lo que apoya la creencia que hay factores externos que impiden su recuperación. Tales factores podrían ser de origen antrópico, como la mortalidad en redes pesqueras o causadas por colisiones con botes, como lo demuestran las necropsias de animales encontrados muertos en esta zona, entre los que hay una alta proporción de animales jóvenes (Félix, Haase, Denkinger, \& Falconí, 2011). En general, los animales jóvenes tienen mayor riesgo de mortalidad debido a su inexperiencia frente a factores naturales como depredación y agresión intraespecífica (e.g., Dunn, Barco, Pabst, \& MacLellan, 2002) y ante factores antrópicos como la interacción con artes de pesca y colisiones con embarcaciones (Wells, Hofmann, \& Moors, 1998; Mann, Scott, \& Smuts, 1995). En otras partes del golfo de Guayaquil se ha encontrado que los animales inmaduros recién destetados tienen un mayor riesgo de quedar atrapados en artes de pesca (Félix et al., 2018a; Félix, Vásconez, Centeno, \& Romero, 2019). Una alta mortalidad de animales jóvenes limitaría la recuperación de la población y reduciría el recambio generacional produciendo un envejecimiento de la población. La madurez sexual tardía y la baja tasa de reproducción de la especie (Wells et al., 1987) son también factores que complican la recuperación poblacional, así como la eventual pérdida de diversidad genética por endogamia (Frère et al., 2010).

La confluencia de diferentes factores antrópicos como la pesca, el tráfico marítimo y la contaminación podrían estar relacionados también con el patrón actual de distribución de delfines de Santa Elena, provocando por ejemplo un concentración de actividades en áreas menos alteradas o incluso la emigración hacia sitios con mejores condiciones ecológicas para alejarse de tales presiones (e.g. Tezanos-Pinto et al., 2013). El alto tráfico de embarcaciones en la parte noroccidental (sector 2), particularmente de embarcaciones pesqueras artesanales que cruzan muy cerca de la costa de la puntilla al salir e ingresar a puerto, explicaría por qué es la zona menos usada por los delfines actualmente, y en su lugar éstos concentran sus actividades en la parte sur de la puntilla 
(sectores 3 y 4), donde no hay ningún puerto y el tráfico de embarcaciones es muy bajo debido al fuerte oleaje. Se ha demostrado que la especie es muy sensible al tráfico marítimo y puede causar el desplazamiento de los animales hacia otras áreas para evitarlo (véase Novacek, Wells, \& Solow, 2001; Lusseau, 2004; Lusseau et al., 2006; Morteo et al., 2012a; Rako et al., 2013; Hernández et al., 2015). La información disponible sugiere que los delfines de Santa Elena han cambiado su distribución y la forma de uso de su hábitat en los últimos años, pues entre 2006 y 2009 hubo siete registros oportunistas en la bahía de Salinas, pero ninguno en los últimos siete años, aunque si hubo uno más al norte (Punta Blanca) en 2016. Así mismo, se ha encontrado en otros sitios una vinculación estrecha entre la presión de pesca y el abandono de áreas tradicionales de los delfines (e.g. Morteo et al., 2012a; Di Giacomo \& Ott, 2016). La alta tasa de cicatrices en el cuerpo de los delfines de Santa Elena reportada recientemente muestra que las colisiones y las redes pesqueras han afectado más a estos animales que en otros sitios del país (Félix et al., 2018a).

El pequeño tamaño poblacional de esta comunidad de delfines y las condiciones oceanográficas rigurosas en la parte sur del área de estudio donde actualmente concentran sus actividades (Mar Bravo-Punta Carnero), son sin duda barreras que dificultan su seguimiento. Creemos que, aunque la tasa de encuentro fue más baja en los monitoreos en carro que desde un bote (promedio 0.081 y 0.15 delfines $/ \mathrm{km}$ de recorrido, respectivamente) debido principalmente a que el tamaño grupal fue subestimado $32 \%$ desde tierra, en términos de costo efectividad el monitoreo desde la costa parece una buena alternativa de bajo costo para monitorear esta población en el largo plazo, que debe ser complementado con monitoreos en bote.

La designación del área circundante a la puntilla de Santa Elena como área protegida en 2008 (MAE, 2012) representa una oportunidad para la conservación y la recuperación de esta población. No hay otra especie marina residente en la zona que requiera mayores esfuerzos de conservación dado el reducido número de individuos de esta comunidad y los factores de riesgo mencionados. El nuevo Código Orgánico del Ambiente ${ }^{1}$ ecuatoriano, que entró en vigor en abril de 2018, otorga al Ministerio de Ambiente la potestad de regular las actividades dentro de las áreas protegidas, incluyendo la pesca (Arts. 37 y 41). Para contrarrestar el impacto de las actividades pesqueras y del tráfico marítimo sugerimos a quienes tienen a su cargo la gestión de la Reserva Puntilla de Santa Elena, tomar las siguientes medidas de ordenamiento:

1. Erradicar de las aguas de la reserva las redes robaleras cambiándolas por artes de pesca más selectivas como palangres como medida precautoria, pues, aunque no se encontraron animales atrapados en estas redes durante el estudio si ha habido registros de delfines muertos en la playa por estas causas (ver Félix et al., 2011). Además, hay una evidente sobreposición entre delfines y faenas pesqueras en la zona de Mar Bravo. La mortalidad incidental de mamíferos marinos en redes es la principal causa de reducción poblacional o limitante de la recuperación de las poblaciones silvestres alrededor del mundo (Reeves et al., 2013).

2. Establecer una franja de exclusión de tráfico marítimo de $1 \mathrm{~km}$ a lo largo de toda la zona costera de la Reserva Puntilla de Santa Elena; este pequeño cambio no representa costos adicionales mayores para los pescadores y reduce considerablemente la posibilidad de colisiones con delfines.

3. Limitar la velocidad de todo tipo de embarcaciones a 10 nudos en la zona de reserva. Se ha demostrado que hay una reducción significativa de las colisiones de barcos con ballenas cuando la velocidad es de 10 nudos o menos (Conn \& Silber, 2013). Esta medida beneficiará directamente a los delfines y también a otras especies como

1. Registro Oficial Año IV No 983, 12 de abril de 2017. Suplemento. Quito, Ecuador. 
tortugas marinas y a las ballenas jorobadas que frecuentan la zona, en particular a las madres con crías que se distribuyen en la zona más costera (Félix \& Botero, 2011).

Reconocemos que tomar medidas restrictivas es difícil por la resistencia que podrían poner algunos actores locales. Por ello las autoridades deberán implementar una gestión precautoria y proactiva, considerando tanto aspectos socioeconómicos como ecológicos. El Código Orgánico del Ambiente también establece que la administración de las áreas protegidas debe realizarse con la participación de los actores locales (Art. 48). Dada la cantidad de actividades sectoriales en la zona, se debe iniciar una estrategia para involucrarlos y utilizar herramientas de planificación y zonificación de la zona marina (e.g. Ehler \& Douvere, 2009), definiendo usos del espacio marino tomando en cuenta su compatibilidad con la conservación del bufeo costero. Debe considerarse además hacer un seguimiento para evaluar la efectividad de las medidas a ser tomadas con horizontes de corto, mediano y largo plazo, y hacer los ajustes del caso. Finalmente recomendamos crear un programa de monitoreo de esta comunidad de delfines para entender mejor su dinámica poblacional, el flujo genético con otras comunidades vecinas e identificar todas las posibles causas de estrés e impactos para la población, complementando otros esfuerzos en marcha en el golfo de Guayaquil (e.g. BayasRea et al., 2018; Félix et al., 2018a).

Declaración de ética: los autores declaran que todos están de acuerdo con esta publicación y que han hecho aportes que justifican su autoría; que no hay conflicto de interés de ningún tipo; y que han cumplido con todos los requisitos y procedimientos éticos y legales pertinentes. Todas las fuentes de financiamiento se detallan plena y claramente en la sección de agradecimientos. El respectivo documento legal firmado se encuentra en los archivos de la revista.

\section{AGRADECIMIENTOS}

Los autores agradecen a operadores turísticos locales que llevaron a bordo a los investigadores y voluntarios que recolectaron datos desde embarcaciones de observación de turismo en los primeros años de estudio. El estudio fue parte de la tesis de grado de Melanie Zavala. Los autores agradecen a cuatro revisores anónimos que hicieron valiosos comentarios para mejorar el artículo. Esta investigación se realizó bajo los permisos de investigación No005-16 IC-FAU-DPSE-MA, No008-16 ICFAU-DPSE-MA y No001-17 IC-FAU-DPSEMA, otorgados por la Dirección de Ambiente de Santa Elena, Ecuador.

\section{RESUMEN}

Una comunidad residente de bufeos costeros Tursiops truncatus ha sido estudiada de manera intermitente entre 2005 y 2018 en la punta de la península de Santa Elena, Ecuador $\left(2^{\circ} 11^{\prime} \mathrm{S} \& 81^{\circ} 0.7^{\prime} \mathrm{W}\right)$. Avistamientos oportunistas y dirigidos desde la playa y abordo de un bote con motor fuera de borda se realizaron a lo largo de $40 \mathrm{~km}$ de costa, contabilizándose un esfuerzo de $917.2 \mathrm{~km}$ de seguimiento en automóvil desde tierra y $707.4 \mathrm{~km}$ en bote por mar. El tamaño grupal promedio fue 5.31 delfines/grupo $(\mathrm{SD}=$ 1.97, rango 1-10), sin cambios significativos a lo largo del estudio. Desde tierra el tamaño grupal fue subestimado en promedio en $32 \%$. Esta comunidad de bufeos tiene actualmente solo nueve individuos, incluyendo seis adultos, un inmaduro y dos crías, siendo la comunidad más pequeña encontrada en el golfo de Guayaquil. La tasa de encuentro varió entre 0.03 delfines $/ \mathrm{km}$ en la parte noroeste y 0.31 delfines $/ \mathrm{km}$ en la parte sur, donde los delfines concentran sus actividades, posiblemente porque las actividades humanas ahí son menos intensas. Los delfines se distribuyeron generalmente entre los primeros $200 \mathrm{~m}$ de la orilla llegando hasta $1200 \mathrm{~m}$ en la parte norte donde la profundidad es menor y las actividades portuaria y turística más intensas. La velocidad de los delfines fue significativamente más alta cuando fueron seguidos desde un bote a corta distancia $(5.87 \mathrm{~km} / \mathrm{h})$ que cuando fueron monitoreados desde tierra $(2.9 \mathrm{~km} / \mathrm{h})(\mathrm{P}<0.01)$, lo cual sugiere que el seguimiento en bote tuvo un impacto sobre el movimiento de los delfines. Un análisis clúster jerárquico mostró que los delfines tienen altos índices de asociación entre ellos (promedio 0.67, rango 0.01-1.0), indicando que es una comunidad altamente cohesionada. Adicionalmente muestran un alto nivel de residencia (índice de ocurrencia promedio de 0.62). Durante el estudio se identificaron dos amenazas principales, una pesquería con redes agalleras en la parte suroeste y un intenso tráfico de botes pesqueros en la parte 
noroeste. La mayoría del área de estudio actualmente es parte de un área costero-marina protegida creada en 2008, lo cual ofrece una oportunidad para la recuperación de esta comunidad de delfines. Debido a su fragilidad, se recomienda a las autoridades ambientales, entre otras cosas, eliminar potenciales fuentes de amenaza para los delfines como las redes de pesca, implementar un área de exclusión para artes de pesca y tráfico de botes pesqueros de $1 \mathrm{~km}$ de ancho desde la orilla, y limitar la velocidad de todo tipo de embarcación a 10 nudos dentro del área protegida.

Palabras clave: ecología; conservación y manejo; delfín costero; Tursiops truncatus; amenazas antrópicas; Ecuador.

\section{REFERENCIAS}

Azevedo, A. F., Carvalho, R. R., Kajin, M., Van Sluys, M., Bisi, T.L., Cunha, H. A., \& Lailson-Brito, J. (2017). The first confirmed decline of a delphinid population from Brazilian waters: 2000-2015 abundance of Sotalia guianensis in Guanabara Bay, South-eastern Brazil. Ecological Indicators, 79, 1-10. DOI: 10.1016/j. ecolind.2017.03.045

Bayas-Rea, R., Félix, F., \& Montúfar, R. (2018). Genetic divergence and fine scale population structure of the common bottlenose dolphin (Tursiops truncatus, Montagu) found in the Gulf of Guayaquil, Ecuador. PeerJ6: e4589. DOI: 10.7717/peerj.4589

Bigg, M. A., Olesiuk, P. F., Ellis, G. M., Ford, J. K. B., \& Balcomb, K. C. (1990). Social organization and genealogy of resident killer whales (Orcinus orca) in the coastal waters of British Columbia and Washington State. Reports of the International Whaling Commission, Special Issue, 12, 383-405.

Cairns, S. J., \& Schwager, S. J. (1987). A comparison of association indices. Animal Behaviour, 35, 1454-1469.

Conn, P. B., \& Silber, G. K. (2013). Vessel speed restrictions reduce risk of collision-related mortality for North Atlantic right whales. Ecosphere, 4(4), 1-15.

Connor, R. C., Smolker, R. A., \& Richards, A. F. (1992). Dolphin alliances and coalitions. In A. M. Marcourt \& F. B. M. de Waal (Eds.), Coalitions and alliances in humans and other animals (pp. 415-443). Oxford. UK: Science Publications.

Di Giacomo, A. B., \& Ott, P. H. (2016). Long-term site fidelity and residency patterns of bottlenose dolphins (Tursiops truncatus) in the Tramandaí Estuary, southern Brazil. Latin American Journal of Aquatic Mammals, 11(1-2), 155-161.

Dungan, S., Wang, J. Y., Araújo, C. C., Yang, S. C., \& White, B. N. (2016). Social structure in a critically endangered Indo-Pacific humpback dolphin (Sousa chinensis) population. Aquatic Conservation: Marine and
Freshwater Ecosystems, 26, 517-529. DOI: 10.1002/ aqc. 2562

Dunn, D. G., Barco, S. G., Pabst, D. A., \& MacLellan, W. A. (2002). Evidence for infanticide in bottlenose dolphins of western north Atlantic. Journal of Wildlife Diseases, $38(2), 505-510$.

Ehler, C., \& Douvere, F. (2009). Marine spatial planning: A step-by-step approach toward ecosystem-based management. IOC Manual and Guides, 53, 1-98.

Félix, F. (1994). Ecology of the coastal bottlenose dolphin Tursiops truncatus in the Gulf of Guayaquil, Ecuador. In G. Pilleri. Investigations on Cetacea, 25, 235-256.

Félix, F. (1997). Organization and social structure of the bottlenose dolphin Tursiops truncatus in the Gulf of Guayaquil, Ecuador. Aquatic Mammals, 23(1), 1-16.

Félix, F., \& Botero, N. (2011). Distribution and behaviour of humpback whales mother/calf pairs during the breeding season off Ecuador. Marine Ecology Progress Series, 426, 277-287.

Félix, F., Calderón, A., Vintimilla, M., \& Bayas-Rea, R. A. (2017). Decreasing population trend in coastal bottlenose dolphin (Tursiops truncatus) from the Gulf of Guayaquil, Ecuador. Aquatic Conservation: Marine and Freshwater Ecosystems. DOI: 10.1002/aqc.2763

Félix, F., Centeno, R., Romero, J., Zavala, M., \& Vásconez, O. (2018a). Prevalence of scars from anthropogenic origin in coastal bottlenose dolphin in Ecuador. Journal of the Marine Biological Association of the United Kingdom, 98(5):1177-1186. DOI: 10.1017/ S0025315417000686

Félix, F., Haase, B., Denkinger, J., \& Falconí, J. (2011). Varamientos de mamíferos marinos registrados en la costa continental de Ecuador entre 1996 y 2009. Acta Oceanográfica del Pacífico, 16(1), 61-73.

Félix, F., Van Waerebeek, K., Sanino, G. P., Castro, C., Van Bressem, M. F., \& Santillán, L. (2018b). Variation in dorsal fin morphology in common bottlenose dolphin Tursiops truncatus (Cetacea: Delphinidae) populations from the Southeast Pacific Ocean. Pacific Science, 72(3), 307-320.

Félix, F., Vásconez, O., Centeno, R., \& Romero, J. (2019). Tough life: the case of a young coastal common bottlenose dolphin repeatedly entangled. Latin American Journal of Aquatic Mammals (LAJAM), 13(1-2), 9-14. DOI: $10.5597 /$ lajam00243

Frère, C. L., Krutzen, M., Kopps, A. M., Ward, P., Mann, J., \& Sherwin, W. B. (2010). Inbreeding tolerance and fitness costs in wild bottlenose dolphins. Proceedings of the Royal Society B, 277, 2667-2673. DOI: 10.1098/rspb.2010.0039

Fruet, P. F., Laporta, P., Di Tullio, J. C., Secchi, E. R., Morteo, E., Tezanos-Pinto, G., ... Palacios, P. (2016). 
Introduction to the Special Volume on Tursiops in the Southwest Atlantic Ocean. Latin American Journal of Aquatic Mammals, 11(1-2), 1-15.

Fury, C. A, Rucksthul, K. E., \& Harrison, P. L. (2013). Spacial and social sexual segregation patterns in Indo-Pacific bottlenose dolphins (Tursiops aduncus). PLoSONE, 8(1), e52987. DOI: 10.1371/journal. pone. 0052987

Hammond, P. S., Bearzi, G., Bjørge, A., Forney, K. A., Karkzmarski, L., Kasuya, T., ... Wilson, B. (2012a). Tursiops truncatus. The IUCN Red List of Threatened Species. 2012:e.T22563A17347397. DOI: 10.2305/ IUCN.UK.2012.RLTS.T22563A17347397.en

Hammond, P. S., Bearzi, G., Bjørge, A., Forney, K. A., Karkzmarski, L., Kasuya, T., ... Wilson, B. (2012b). Tursiops aduncus. The IUCN Red List of Threatened Species 2012:e.T41714A17600466. DOI: 10.2305/ IUCN.UK.2012.RLTS.T41714A17600466.en

Hernández, I., Morteo, E., Heckel, G., Sosa, O., Álvarez, G., Flores, O., \& Martínez-Serrano, I. (2015). Caracterización de la distribución espacio-temporal de los tursiones (Tursiops truncatus) y las actividades humanas en el Parque Nacional Sistema Arrecifal Veracruzano. E-BIOS, 2(8), 34-52.

Herrera, J. C., Capella, J., Soler, G., Bessudo, S., García, C., \& Flórez-González, L. (2011). Ocurrencia y tasas de encuentro de mamíferos marinos en las aguas de la isla Malpelo y hacia el continente. Boletín de Investigaciones Marinas y Costeras INVEMAR, 40, 57-78.

Herrera, M., Castro, R., Coello, D., Saa, I., \& Elías, E. (2013). Ports, coves and artisanal fishing settlements on mainland Ecuador. Instituto Nacional de Pesca, Ecuador. Boletín Especial, 4(1), 1-616.

Hurtado, M., Hurtado, M., \& Hurtado, L. (2012). Estado de la contaminación marina en Ecuador. Informe de Consultoría a la Comisión Permanente el Pacífico Sur (CPPS). Guayaquil, Ecuador.

Instituto Nacional de Estadísticas y Censo (INEC). (2017). Fascículo provincial Santa Elena. Instituto Nacional de Estadísticas y Censo. Recuperado de http://www.ecuadorencifras.gob.ec/ wp-content/descargas/Manu-lateral/Resultados-provinciales/santa_elena.pdf

Instituto NAZCA de Investigaciones Marinas. (2008). Monitoreo ecológico y levantamiento de cartografía marina de la Puntilla de Santa Elena. Estudio previo a la declaración como área protegida (Provincia Santa Elena - Cantón Salinas). Informe final al Ministerio de Ambiente de Ecuador, Quito, Ecuador.

Jefferson, T. A., Webber, M. A., \& Pitman, R. L. (2008). Marine mammals of the world, a comprehensive guide to their identification. London, UK: Academic Press.
Jiménez, P., Alava, J. J., Castro, C., Denkinger, J., \& Haase, B. (2011). Bottlenose dolphin (Tursiops truncatus). In D. G. Tirira (Ed.), Libro rojo de los mamíferos del Ecuador (pp. 229-230). Ecuador: Fundación Mamíferos y Conservación. Pontificia Universidad Católica del Ecuador y Ministerio del Ambiente del Ecuador.

Lusseau, D. (2004). The hidden cost of tourism: Detecting long-term effects of tourism using behavioral information. Ecology and Society, 9(1), 2.

Lusseau, D., Wilson, B., Hammond, P. S., Grellier, K., Durban, J. W., Parsons, K. M., ... Thompson, P. M. (2006). Quantifying the influence of sociality on population structure in bottlenose dolphins. Journal of Animal Ecology, 75, 14-24. DOI: 10.1111/j.1365-2656.2005.01013.x

Mann, J., Scott, R. A., \& Smuts, B. B. (1995). Responses to calf entanglement in free-ranging bottlenose dolphins. Marine Mammal Science, 11(1), 100-106.

Ministerio de Ambiente de Ecuador (MAE). (2012). Plan de manejo de la Reserva de Producción Faunística Marino Costera Puntilla de Santa Elena. Ministerio de Ambiente, Quito.

Möller, L. M., Wiszniewski, J., Allen, S. J., \& Beheregaray, B. (2007). Habitat type promotes rapid and extremely localized genetic differentiation in dolphins. Marine and Freshwater Research, 58, 640-648.

Morteo, E., Rocha-Olivares, A., \& Abarca-Arenas, L. G. (2014). Sexual segregation in coastal bottlenose dolphins (Tursiops truncatus) in the south-western Gulf of Mexico. Aquatic Mammals, 40(4), 375-385. DOI: 10.1578/AM.40.4.2014.375

Morteo, E., Rocha-Olivares, A., Arceo-Briseño, P., \& Abarca-Arenas, L. G. (2012a). Spatial analyses of bottlenose dolphin-fisheries interactions reveal human avoidance off a productive lagoon in the western Gulf of Mexico. Journal of the Marine Biological Association of the United Kingdom, 92(8), 1893-1900. DOI: $10.1017 / \mathrm{S} 0025315411000488$

Morteo, E., Rocha-Olivares A., \& Morteo, R. (2012b). Sensitivity analysis of residency and site fidelity estimations to variations in sampling effort and individual catchability. Revista Mexicana de Biodiversidad, 83, 487-495.

Natoli, A., Peddemors, V. M., \& Hoelzel, A. R. (2004). Population structure and speciation in the genus Tursiops based on microsatellite and mitochondrial DNA analyses. Journal of Evolutionary Biology, 17(2), 363-375. DOI: 10.1046/j.1420-9101.2003.00672.x

Novacek, S. M., Wells, R., \& Solow, A. R. (2001). Shortterm effects of boat traffic on bottlenose dolphins, Tursiops truncatus, in Sarasota Bay, Florida. Marine Mammal Science, 17, 663-688. 
Olavarría, C., Acevedo, J., Vester, H., Zamorano-Abramson, J., Vidi, F., Gibbons, J., ... Torre-Flórez, J. P. (2010). Southernmost distribution of common bottlenose dolphins (Tursiops truncatus) in the Eastern South Pacific. Aquatic Mammals, 36(3), 288-293.

Parsons, E. C. M., \& Jefferson, T. A. (2000). Post-mortem investigations on stranded dolphins and porpoises from Hong Kong waters. Journal of Wildlife Diseases, 36(2), 342-356. DOI: 10.7589/0090-3558-36.2.342

Perrin, W. F., Thieleking, J. L., Walter, W. A., Archer, F. I., \& Robertson, K. M. (2011). Common bottlenose dolphins (Tursiops truncatus) in California waters, cranial differentiation of coastal and offshore ecotypes. Marine Mammal Science, 27(4), 769-792. DOI: 10.1111/j.1748-7692.2010.00442.x

QGIS Development Team. (2016). QGIS Geographic Information System. Open Source Geoespatial Foundation Project. Recuperado de http//qgis.osgeo.org

Rako, N., Fortuna, C. M., Holcer, D., Mackelworth, P., Nimak-Wood, M., Pleslic, G., ... Picciulin, M. (2013). Leisure boating noise as a trigger for the displacement of the bottlenose dolphins of the CresLosinj archipelago (northern Adriatic Sea, Croatia). Marine Pollution Bulletin, 68, 77-84. DOI: 10.1016/j. marpolbul.2012.12.019

Reeves, R. R., McClellan, K., \& Werner, T. (2013). Marine mammal bycatch in gillnet and other entangling net fisheries, 1990 to 2011. Endangered Species Research, 20, 71-97. DOI: 10.3354/esr00481

Reeves, R. R., Smith, B. D., Crespo, E. A., \& Notarbartolo di Sciara, G. (compilers) (2003). Dolphins, whales and porpoises, 2002-2010 Conservation Action Plan for the World's Cetaceans. Gland, Switzerland and Cambridge, UK: IUCN/SSC Cetacean Specialist Group.

Sanino, G., Van Waerebeek, K., Van Bressem, M. F., \& Pastene, L. A. (2005). Preliminary note on population structure in Eastern South Pacific common bottlenose dolphins, Tursiops truncatus. Journal of Cetacean Research and Management, 7, 65-70.

Shane, S. H. (1990). Behavior and ecology of the bottlenose dolphin at Sanibel Island, Florida. In S. Leatherwood \& R. R. Reeves (Eds.), The bottlenose dolphin (pp. 245-265). San Diego, USA: Academic Press.

Simões-Lopes, P. C., \& Fabian, M. E. (1999). Residence patterns and site fidelity in bottlenose dolphins, Tursiops truncatus (Montagu) (Cetacea, Delphinidae) off Southern Brazil. Revista Brasileira de Zoologia, 16(4), 1017-1024.
Stevenson, M. (1981). Seasonal variations in the Gulf of Guayaquil, a tropical estuary. Boletín Cientifico $y$ Técnico, 4, 5-133.

Tezanos-Pinto, G., Baker, C. S., Russell, K., Martien, K., Baird, R. W., Hutt, A. ... Garrige. C. (2009). A worldwide perspective on the population structure and genetic diversity of bottlenose dolphins (Tursiops truncatus) in New Zealand. Journal of Heredity, 100(1), 11-24.

Tezanos-Pinto, G., Constantine, R., Brooks, L., Jackson, J. A., Mourao, F., Wells, S., \& Baker, S. (2013). Decline in local abundance of botlenose dolphins (Tursiops truncatus) in the Bay of Islands, New Zealand. Marine Mammal Science, 29(4), E390-E410. DOI: 10.1111/mms. 12008

Van Bressem, M., Simoes-Lopes, P. C., Félix, F., Kiszka, J., Daura-Jorge, F. G., Avila, I. C., ... Van Waerebeek, K. (2015). Epidemiology of lobomycosis-like disease in bottlenose dolphins (Tursiops spp.) from South America and southern Africa. Diseases of Aquatic Organisms, 117, 59-75. DOI: 10.3354/dao02932

Van Waerebeek, K., Baker, A. N., Félix, F., Gedamke, J., Iñiguez, M., Sanino, G. P., ... Wang, Y. (2007). Vessel collisions with small cetaceans worldwide and with large whales in the Southern Hemisphere, and initial assessment. Latin American Journal of Aquatic Mammals, 6(1), 43-69.

Van Waerebeek, K., Reyes, J. C., Read, A. J., \& McKinnon, J. S. (1990). Preliminary observations of bottlenose dolphins from the Pacific coast of South America. In S. Leatherwood \& R. R. Reeves (Eds.), The bottlenose dolphin (pp. 143-154). San Diego, USA: Academic Press.

Van Waerebeek, K., Reyes, J. C., Sanino, G. P., Félix, F., Van Bressem, M. F., Ávila, I. C., ...Venegas, A. (2017, mayo). Common bottlenose dolphins Tursiops truncatus of Pacific South America, a synoptic review of population identification data $(\mathrm{SC} / 67 \mathrm{~A} / \mathrm{SM} / 10)$. Symposium conducted at the meeting of $67^{\text {th }}$ Scientific Committee of the International Whaling Commission, Bled, Slovenia.

Wells, S. R., Hofmann, S., \& Moors, T. (1998). Entanglement and mortality of bottlenose dolphins, Tursiops truncatus, in recreational fishing gear in Florida. Fishery Bulletin, 96(3), 647-650.

Wells, R. S., Scott, M. D., \& Irvine, A. B. (1987). The social structure of free-ranging bottlenose dolphins. In H. H. Genoways (Ed.), Current Mammalogy 1 (pp. 247-305). New York and London: Plenum Press.

Whitehead, H. (2009). SOCPROG programs, Analyzing animal social structures. Behavioral Ecology and Sociobiology, 63, 765-778. 